\title{
SPECKLE INTERFEROMETRY AND IMAGE RECONSTRUCTION
}

Gerd P. Weigelt

Physikalisches Institut, Universität Erlangen-Nürnberg, 8520 Erlangen, E.-Rommel-Str.1, West Germany

\section{$\underline{\text { ABSTRACT }}$}

The angular resolution of conventional astrophotography is limited to about 1 sec of arc due to image degradation by the atmosphere and telescope aberrations. Higher resolution can be achieved by Labeyrie's speckle interferometry, which yields the autocorrelation of astronomical objects with diffraction-limited resolution, e.g. $0.02 \mathrm{sec}$ of arc with $5 \mathrm{~m}$ telescope aperture.

We have investigated modifications of speckle interferometry, which yield diffraction-limited images. We investigated (1) "speckle holography" for image reconstruction of objects near an unresolvable point source (reference star), (2) "speckle masking" for image reconstruction of binary stars (without a reference star), and (3) "phase flipping" for image reconstruction of centro-symmetric objects. Together these three image reconstruction methods represent a set of speckle evaluation procedures, which can yield high resolution images of most astronomical objects that are bright enough for speckle interferometry. Speckle holography was already applied to astronomical objects. We reconstructed real images of the binary star Zeta Cancri $A-B$ and the triple star ADS $3358 \mathrm{~A}-\mathrm{B}-\mathrm{C}(0.07 \mathrm{sec}$ of arc resolution with $1.8 \mathrm{~m}$ telescope aperture). 
1. INTRODUCTION

Speckle interferometry was first proposed

by $A$. Labeyrie ${ }^{1,2}$. Speckle interferometry yields the diffractionlimited autocorrelation only, not a direct image of the object. Therefore many modifications for image reconstruction have been investigated ${ }^{3-11}$.

Our image reconstruction concept consists of the following three methods:

I. "Speckle holography" 3,4,5,6,11 for the reconstruction of objects near an unresolvable star (reference star) (such objects are multiple stars, asteroids or moons or resolvable star disks near an unresolvable star, and possibly some galactic nuclei near an unresolvable star).

II. "Speckle masking" 10 for the reconstruction of binary stars without a reference star (largest class of objects).

III. "Phase flipping" 11 for image reconstruction of centrosymmetric objects (e.g. resolvable star disks and possibly some bright galactic nuclei).

The advantage of the described three methods is (1.) that they can easily be applied and (2.) that most astronomical objects that are bright enough for speckle interferometry (i.e. brighter than about $14^{\mathrm{m}}$ ) can be investigated by at least one of these three methods.

The described image reconstruction methods were already described in earlier publications. Therefore we shall only outline the principle of speckle masking and speckle holography. In section 2 a laboratory experiment illustrating speckle masking is shown. In section 3 we shall sketch the principle of speckle holography and show the first application of speckle holography 
to astronomical objects. In section 4 we shall discuss some limitations of speckle techniques such as isoplanicity, temporal correlation of speckles, resolution and brightness limit.

\section{SPECKLE MASKING}

Speckle masking ${ }^{10}$ is a method, which can yield diffractionlimited images of binary stars instead of autocorrelations. In an image one can measure the position angle without ambiguity, whereas in the autocorrelation there is a $180^{\circ}$-ambiguity. Fig. 1 shows a laboratory experiment illustrating speckle masking. Fig: 1 shows (from the top) the laboratory binary star, one of 3 simulated speckle interferograms, one of 3 new speckle interferograms (obtained from the original speckle interferograms by a masking procedure), one of 3 speckle holograms, and finally the reconstructed image of the binary star. For the application of speckle masking no reference star must exist near the binary star, because the reference star is produced from ordinary binary star speckle interferograms by image processing (masking). For more details see Ref. 10 . 

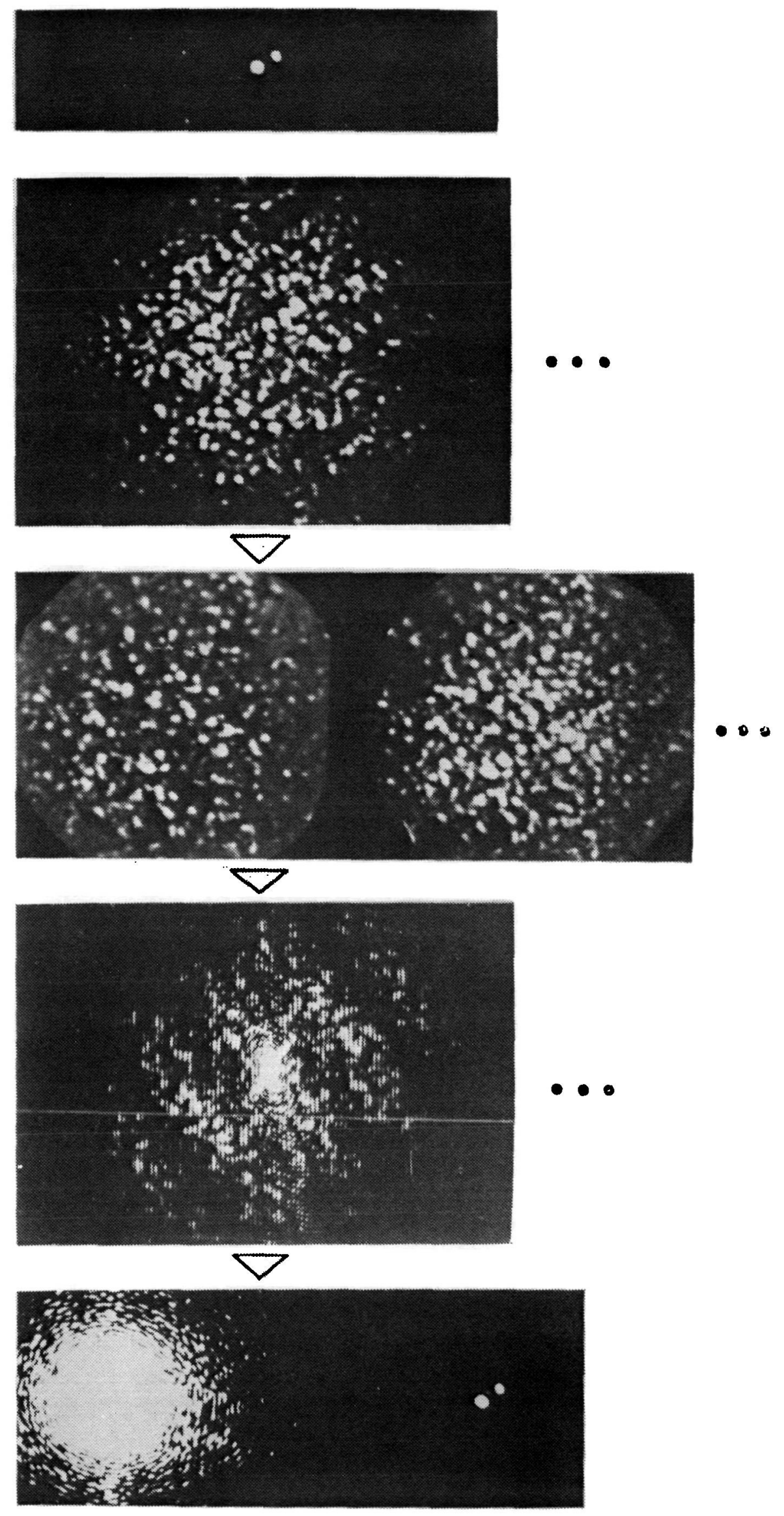

Fig. 1 Laboratory experiment illustrating speckle masking. 
3. SPECKLE HOLOGRAPHY 15

The speckle holography concept $3,4,5,6,11$ consists of (1) a mathematical idea and (2) some image processing techniques.

(1) Mathematical idea: If one wants to reconstruct an image of an object $O(x, y)$, one has to record speckle interferograms of a larger object, which consists of $O(x, y)$ and, additionally, an unresolvable star (reference star) $\delta\left(x-x_{R}, y-y_{R}\right)$ in the isoplanatic patch of $O(x, y)$ (see section 4 for isoplanicity). In other words, one has to record speckle interferograms of the "total" object $O_{T}(x, y)=O(x, y)+\delta\left(x-x_{R}, y-y_{R}\right)$. The speckle interferograms of $O_{T}$ are then processed by the Labeyrie technique to obtain the autocorrelation $\mathrm{O}_{\mathrm{T}} * \mathrm{O}_{\mathrm{T}}$ of the total object $\mathrm{O}_{\mathrm{T}}$ :

$$
\begin{aligned}
\mathrm{O}_{\mathrm{T}} * \mathrm{O}_{\mathrm{T}}=\delta(\mathrm{x}, \mathrm{y})+\mathrm{O}(\mathrm{x}, \mathrm{y}) * \mathrm{O}(\mathrm{x}, \mathrm{y}) & +\mathrm{O}\left(-\mathrm{x}+\mathrm{x}_{\mathrm{R}},-\mathrm{y}+\mathrm{y}_{\mathrm{R}}\right) \\
& +\mathrm{O}\left(\mathrm{x}+\mathrm{x}_{\mathrm{R}}, \mathrm{y}+\mathrm{y}_{\mathrm{R}}\right)
\end{aligned}
$$

Obviously the autocorrelation of $O_{T}$ has a more useful structure than the autocorrelation of $O$, because the autocorrelation of $O_{T}$ contains a real image $O\left(x+x_{R}, y+y_{R}\right)$. The reference star served as decoding key for image reconstruction.

(2) Image processing techniques: If the distance from the reference star to the object $O(x, y)$ is not larger than $3 / 2$ the diameter of $O(x, y)$, then additional image processing techniques are necessary in order to separate $O\left(x+x_{R}, y+y_{R}\right)$ from all other terms in $\mathrm{O}_{\mathrm{T}} * \mathrm{O}_{\mathrm{T}}$. These techniques (brightness variation of the reference star, use of a long exposure photograph and use of different reference stars) are described in Ref. 3 and 6 .

Now we shall describe the application of speckle holography to the binary star Zeta Cancri $A-B$ and the triple star ADS 3358 $A-B-C$ (these experiments are also published in Ref. 12) . Fig. 2 shows the zeta Cancri experiment. Fig. $2 \mathrm{a}$ is one of 40 speckle interferograms of zeta $C N C A-B-C$, which were recorded with the 


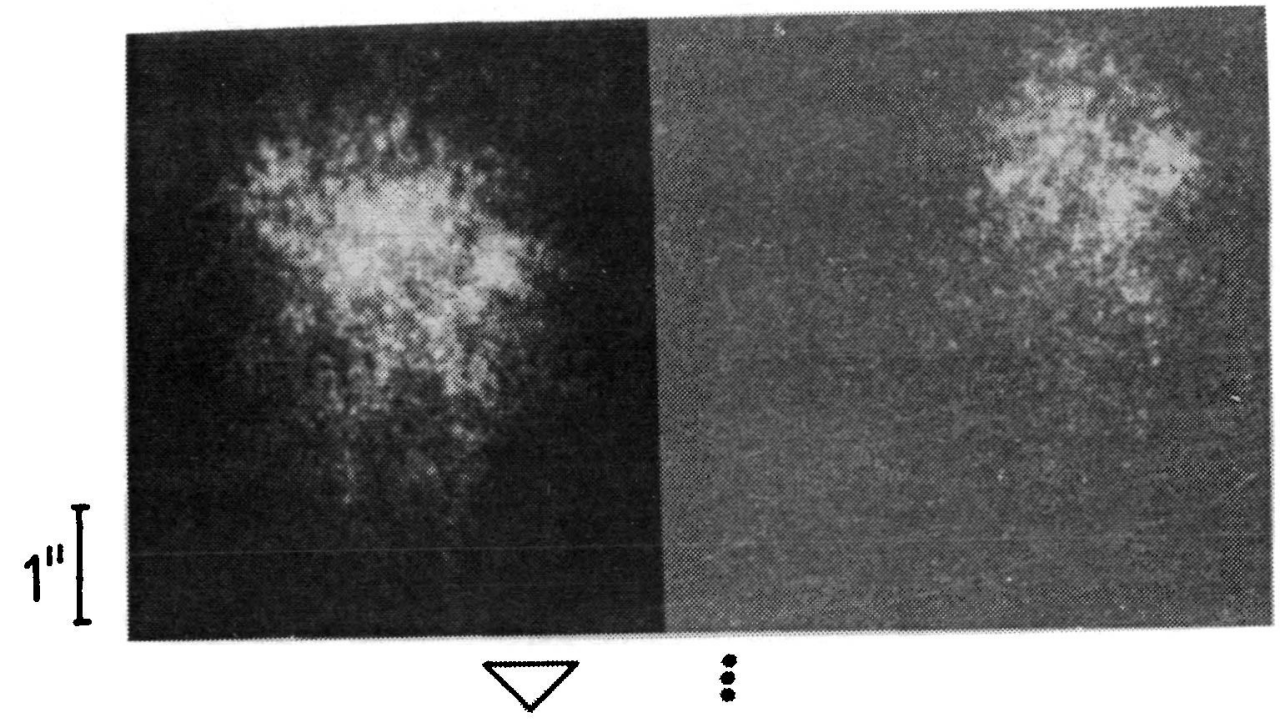

a

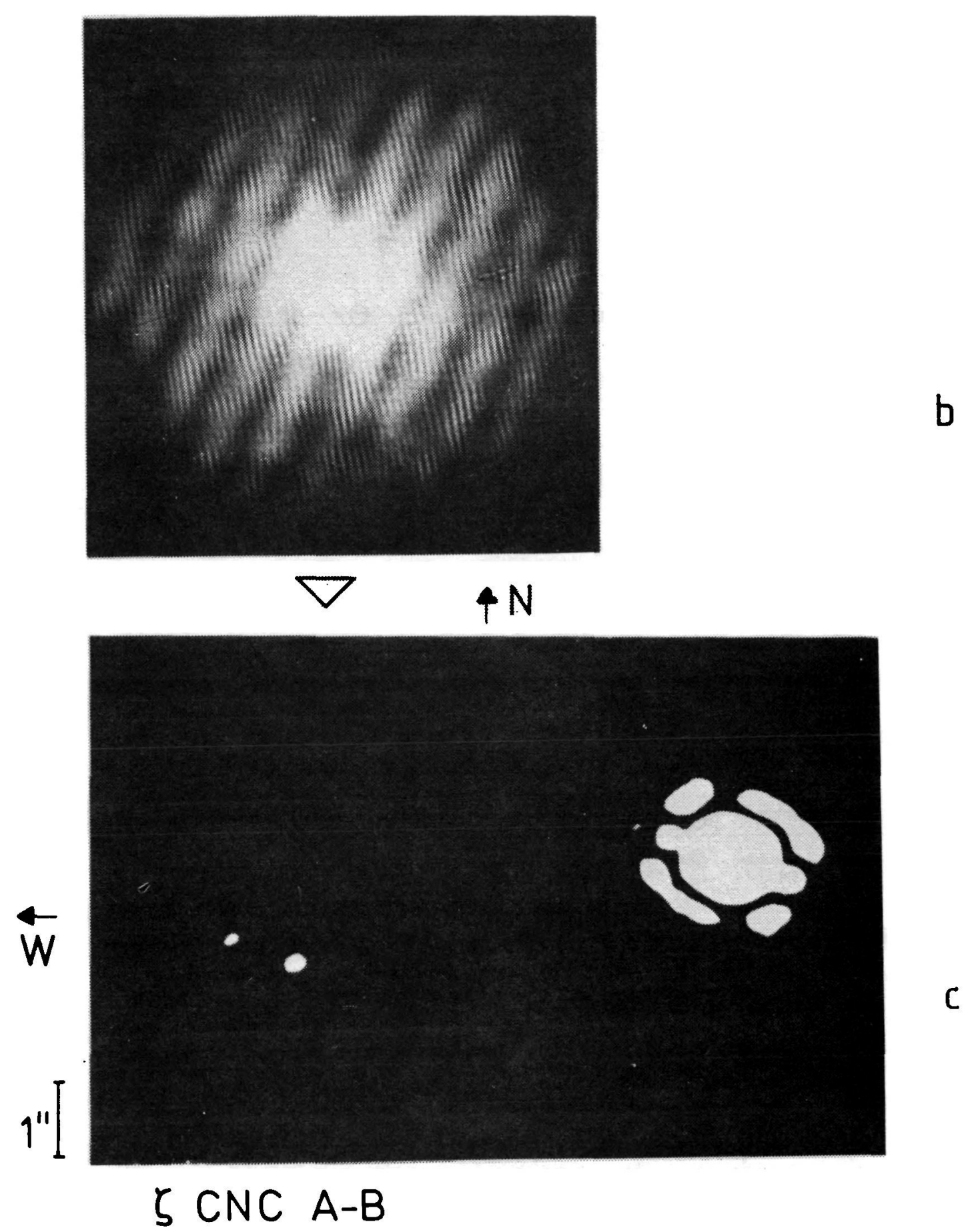

Fig. 2 Speckle holography experiment of Zeta Cancri A-B. From Ref. 12. 
$1 \mathrm{~m}$-telescope of Hoher List observatory. The speckle cloud on the left-hand side of Fig. $2 \mathrm{a}$ is produced by zeta CNC A-B and the other speckle cloud ( $6 \mathrm{sec}$ of arc apart from A-B) is produced by the additionally recorded Zeta $C N C$, which served as reference star. The 40 speckle interferograms were processed optically in order to obtain the average power spectrum (Fig. 2b) and the autocorrelation (Fig. 2c shows one half of the autocorrelation). The autocorrelation contains a real image of zeta CNC A-B. We measured the separation and the position angle of zeta CNC A-B to be $0.86^{\prime \prime} \pm 0.02^{\prime \prime}$ and $291^{\circ} \pm 2^{\circ}(1977.797)$, respectively.

Fig. 3 shows the ADS 3358 experiment. Fig. $3 a$ is one of 240 speckle interferograms of ADS $3358 \mathrm{~A}-\mathrm{B}-\mathrm{C}$, which were recorded with the 1.8m-telescope of Asiago observatory (Italy). Fig. 3b shows the optically produced average power spectrum and Fig. $3 \mathrm{C}$ is the optically reconstructed image of ADS $3358 \mathrm{~A}-\mathrm{B}-\mathrm{C}$. The brightness of the three stars is known to be $5.6^{\mathrm{m}}-7.6^{\mathrm{m}}-7.6^{\mathrm{m}}$. We obtained from our measurements (1977.986): separation of A-B: $0.26 " \pm 0.03^{\prime \prime}$; separation of $\mathrm{A}-\mathrm{C}: 0.88^{\prime \prime} \pm 0.02 "$; position angle of $A-B: 148^{\circ} \pm 3^{\circ}$; position angle of $A-C: 226^{\circ} \pm 2^{\circ}$. The reconstruction of an image of the complete ADS 3358 A-B-C system was possible, because we used two of the stars as reference stars. More precisely, the autocorrelation of the triple system $A-B-C$ (see Fig. 4) contains the crosscorrelation $C * A-B \quad(C=$ reference star) and the crosscorrelation $B * A-C(B=$ reference star $)$. $C * A-B$ represents a sharp image of $A-B$ and $B * A-C$ of $A-C$. Both crosscorrelations yield a sharp image of the complete triple system. 


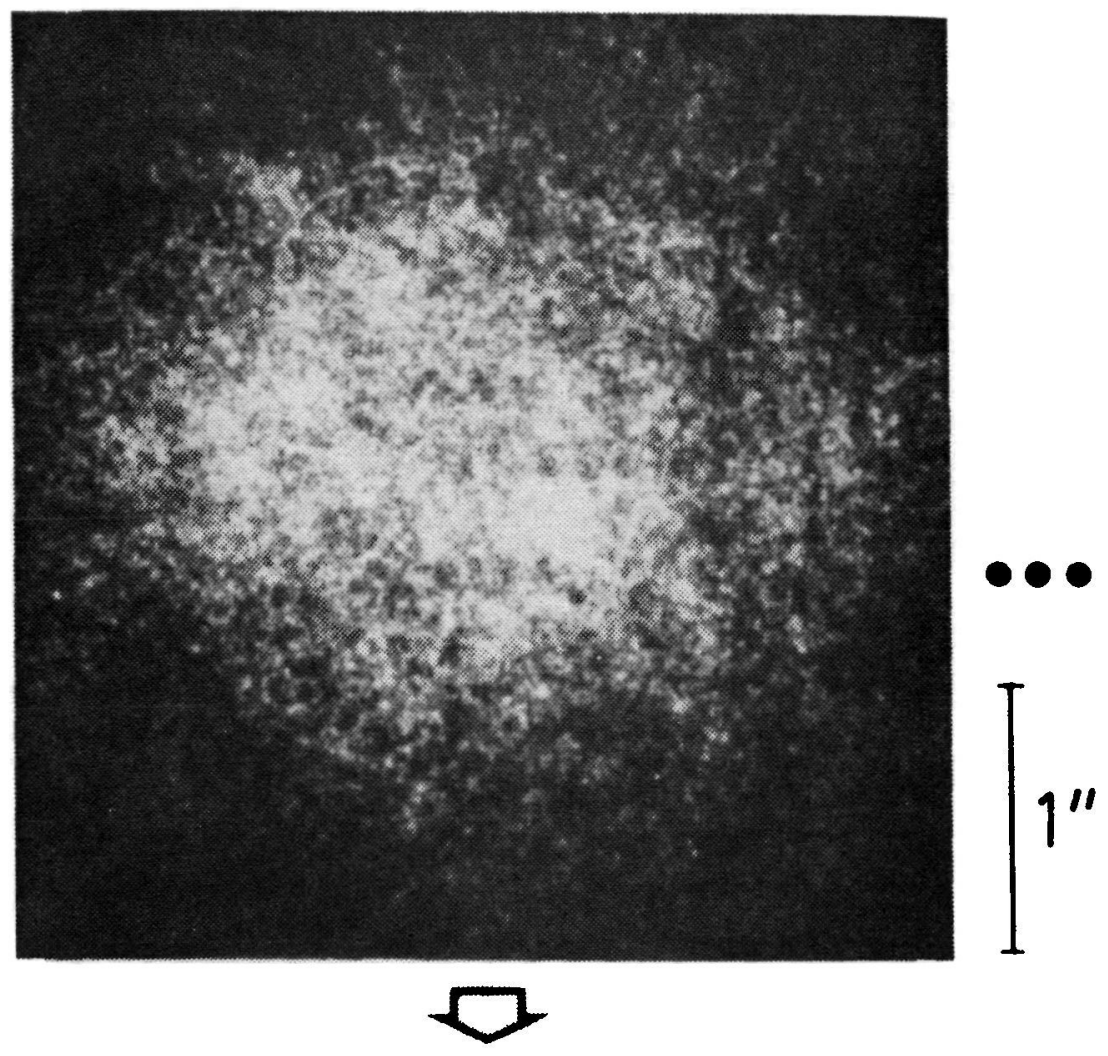

a

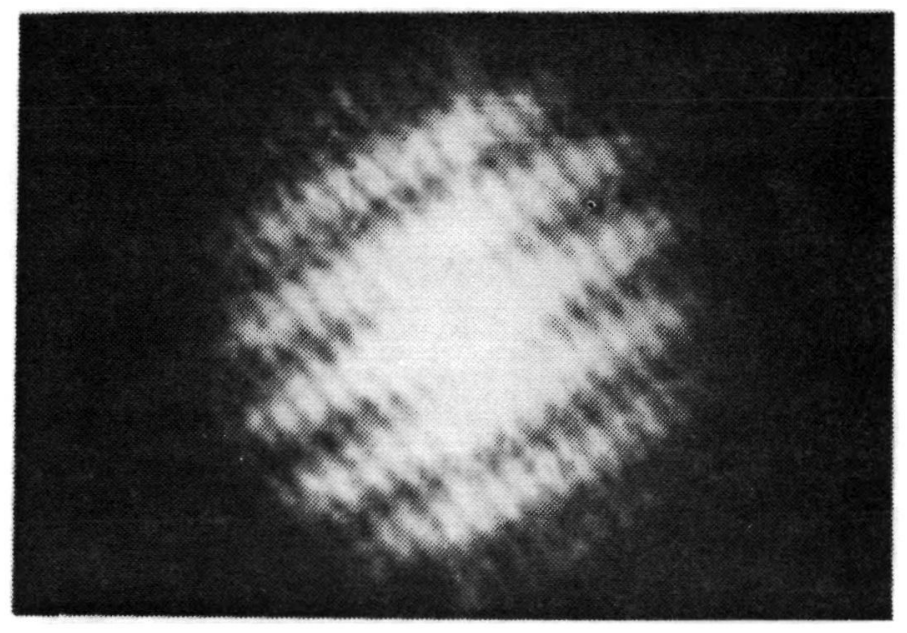

b

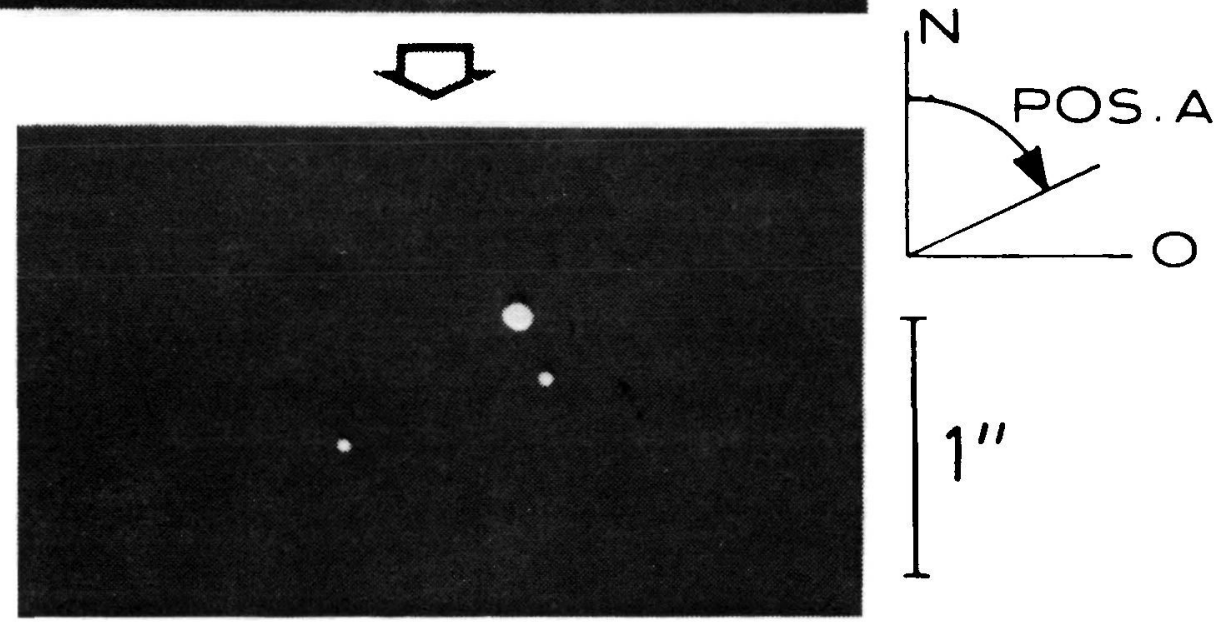

ADS 3358 A-B-C

Fig. 3 Speckle holography experiment of ADS 3358 A-B-C. The photograph at the bottom shows the reconstructed image of the triple star. From Ref. 12. 


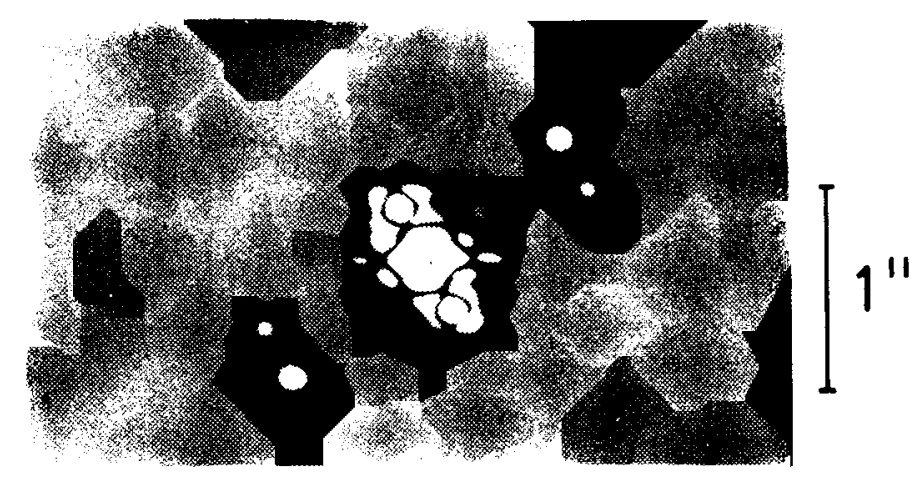

Fig. 4 Optically reconstructed autocorrelation of ADS 3358 .

4. EXPOSURE TIME, RESOLUTION, BRIGHTNESS LIMIT, AND

\section{ISOPLANICITY FOR SPECKLE TECHNIQUES}

Exposure time: We have studied the longest possible exposure time for obtaining high contrast speckles by correlating optically neighborhood speckle interferograms on a $16 \mathrm{~mm}$-motion picture film (recorded at a rate of 64 frames per sec). The correlation time was about $1 / 60$ sec during our measurements (bad seeing; for more details see Ref. 11 and 13).

Resolution: The spectroscopic binary star Alpha AUR (separation $0.04 "$ to $0.06 "$ ") was resolved with a $1.8 \mathrm{~m}$-telescope, although the theoretical resolution (defined as $1.22 \lambda / D$ ) of the telescope used was $0.07 "(\lambda=510 \mathrm{~nm}, \mathrm{D}=1.8 \mathrm{~m})$. For more details see Ref. 14 .

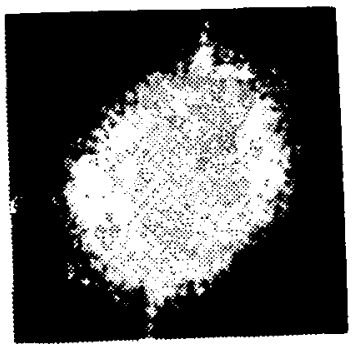

Fig. 5 Average power spectrum of Alpha AUR reconstructed from 40 speckle interferograms recorded with a $1.8 \mathrm{~m}$-telescope. 
Brightness limit: Our faintest resolved binary star was ADS $2630\left(8.9^{\mathrm{m}}-9.3^{\mathrm{m}}\right)$. This object was easily resolved from only 40 speckle interferograms recorded with a $1 \mathrm{~m}$-telescope (Fig. 6; for more details see Ref. 14). Therefore we believe that $12^{\mathrm{m}}$ or $14^{\mathrm{m}}$ can be achieved soon.
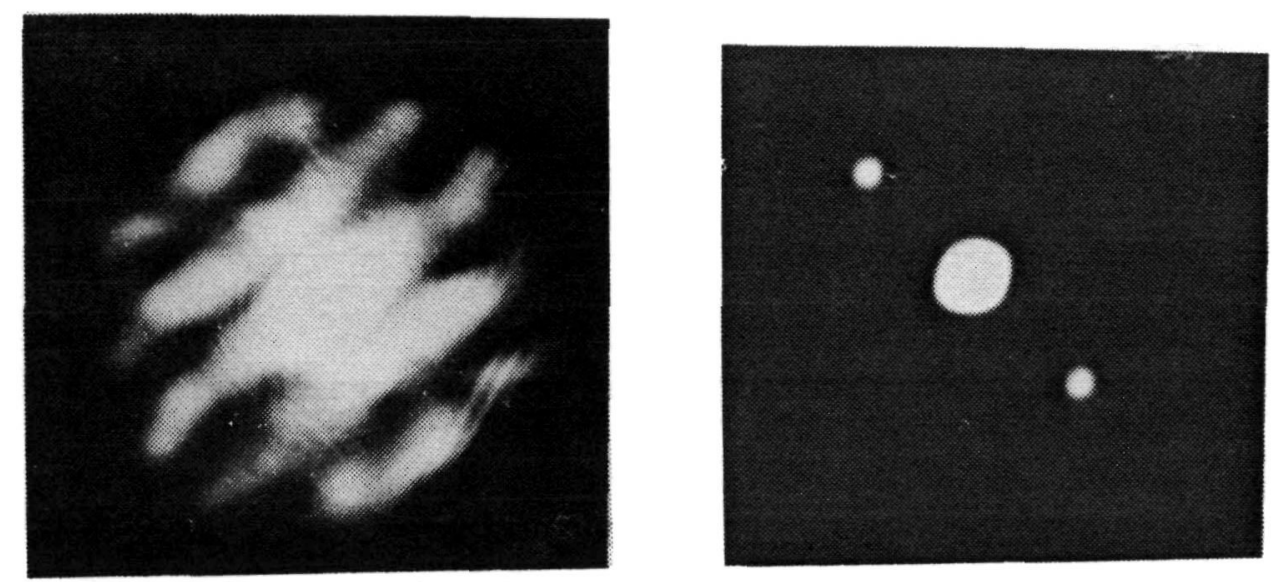

Fig. 6 Powerspectrum and autocorrelation of ADS 2630 (separation $0.55 "$ " 1977.8).

Isoplanicity: The 6"-binary star zeta CNC (Fig.1) and the 10"-binary star Gamma AND (Fig. 7) were measured by speckle interferometry. The diameter of the autocorrelation peaks is about equal to the speckle diameter. Hence, the diameter of the isoplanatic patch was larger than $10 \mathrm{sec}$ of arc during our measurements (for more details see Ref. 13).

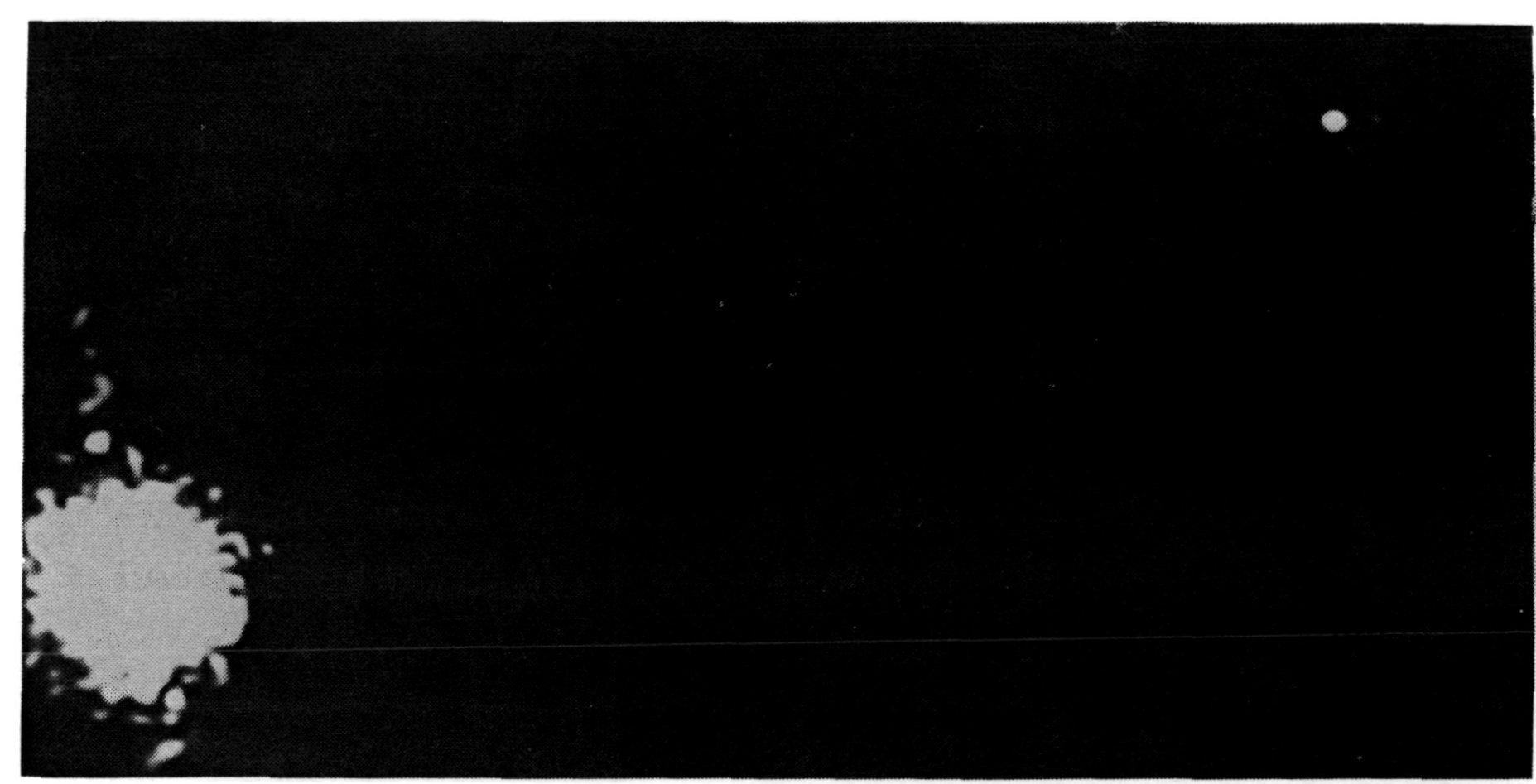


Fig. 7 One half of the autocorrelation of the 10"-binary star Gamma AND reconstructed from 80 speckle interferograms recorded with a $1 \mathrm{~m}$-telescope.

\section{REFERENCES}

1) A. Labeyrie, Astron.Astrophys. 6 (1970) 85

2) D.Y. Gezari, A.Labeyrie, R.V.Stachnik, Astrophys.J.173(1972) L1

3) C.Y.C.Liu, A.W.Lohmann, Opt.Comm. 8 (1973) 372

4) R.H.T.Bates, P.T.Gough, P.J.Napier,Astron.Astrophys. 22 (1973) 319

5) P.T.Gough,R.H.T.Bates, Opt.Acta 21 (1974) 243

6) G.P.Weigelt, Optik 43 (1975) 111

7) K.T.Knox and B.J.Thomson, Astrophys.J. (Letters) 193 (1974) L45

8) C.R.Lynds, S.P.Worden and J.W.Harvey,Astrophys.J.207(1976) 174

9) D.C.Ehn and P.Nisenson, J.Opt.Soc.Am. 65 (1975) 1196

10) G.P.Weigelt, Opt.Comm. 21 (1977) 55

11) A.W.Lohmann and G.P.Weigelt, in Optical Telescopes of the Future. Conf.Proc. (page 479) Dec.77.Geneva:ESOc/OCERN

12) G.P.Weigelt, "Speckle Holography Measurements of the Stars Zeta Cancri and ADS 3358", Appl.Opt., Sept. or Oct. 78

13) A.W.Lohmann and G.P.Weigelt, "Astronomical Speckle Interferometry; Measurements of Isoplanicity and Temporal correlation", submitted to OPTIK

14) G.P.Weigelt, "Speckle Interferometry Measurements of 12 Binary Stars", submitted to Astron.Astrophys.

15) for more details see Ref. 12

\section{DISCUSSION}

M. Miller: At large binary separations, does the autocorrelation function have the correct characteristics to imply partial isoplanicity?

G. Weigelt: The measurement of the 10" binary $\gamma$ And shows that we had partial isoplanicity up to 10". The diameter of 
the correlation peaks was equal to the average speckle diameter, i. e., about 0:15 (a one meter telescope was used).

A. H. Greenaway: In a multiple star system the autocorrelation defines the object but always with rotational ambiguites. In obtaining speckle holographic images how have you resolved such ambiguities?

G. Weigelt: The position angle of the reference star was known from conventional measurements.

D. Y. Gezari: Was the disk of ADS 3358-A resolved in your reconstruction?

G. Weigelt: No, it was not. The disk diameters in the reconstructed image of this triple star indicate the brightnesses of the stars only.

J. C. Dainty: Radioastronomers have several techniques for unravelling multi-point source maps from autocorrelation functions; this was discussed at the IAU Colloquium No. 49 held a few weeks ago at Grongingen (10 - 12 August 1978; proceedings are to be published by Reidel in their Astrophysics and Space Science series). 